Commun. Korean Math. Soc. 27 (2012), No. 3, pp. 547-556

http://dx.doi.org/10.4134/CKMS.2012.27.3.547

\title{
A NEW EXTENSION ON THE HARDY-HILBERT INEQUALITY
}

\author{
Yu Zhou and Mingzhe Gao
}

AbStRaCt. A new Hardy-Hilbert type integral inequality for double series with weights can be established by introducing a parameter $\lambda$ (with $\lambda>1-\frac{2}{p q}$ ) and a weight function of the form $x^{1-\frac{2}{r}}$ (with $r>1$ ). And the constant factors of new inequalities established are proved to be the best possible. In particular, for case $r=2$, a new Hilbert type inequality is obtained. As applications, an equivalent form is considered.

\section{Introduction}

Let $\left\{a_{n}\right\}$ and $\left\{b_{n}\right\}$ be non-negative sequences of real numbers, $\frac{1}{p}+\frac{1}{q}=1$ and $p>1$. If $\sum_{n=1}^{\infty} a_{n}^{p}<+\infty$ and $\sum_{n=1}^{\infty} b_{n}^{q}<+\infty$, then

$$
\sum_{m=1}^{\infty} \sum_{n=1}^{\infty} \frac{\left(\ln \frac{m}{n}\right) a_{m} b_{n}}{m-n} \leq\left(\frac{\pi}{\sin \frac{\pi}{p}}\right)^{2}\left(\sum_{n=1}^{\infty} a_{n}^{p}\right)^{\frac{1}{p}}\left(\sum_{n=1}^{\infty} b_{n}^{q}\right)^{\frac{1}{q}}
$$

and

$$
\sum_{m=1}^{\infty} \sum_{n=1}^{\infty} \frac{a_{m} b_{n}}{m+n} \leq \frac{\pi}{\sin \frac{\pi}{p}}\left(\sum_{n=1}^{\infty} a_{n}^{p}\right)^{\frac{1}{p}}\left(\sum_{n=1}^{\infty} b_{n}^{q}\right)^{\frac{1}{q}},
$$

where the constant factors $\left(\frac{\pi}{\sin \frac{\pi}{p}}\right)^{2}$ in (1.1) and $\frac{\pi}{\sin \frac{\pi}{p}}$ in (1.2) are the best possible. And the equalities in (1.1) and (1.2) hold if and only if $\left\{a_{n}\right\}$, or $\left\{b_{n}\right\}$ is a zero-sequence. They are the famous Hardy-Hilbert inequalities (see [6]),

Owing to the importance of the Hardy-Hilbert inequality in analysis and applications, some mathematicians have been studying them. In particular, some excellent results of (1.2) appear in a lot of the articles (such as $[1,2,3,4,8]$ etc.). However, the research articles of (1.1) are few. The purpose of the present paper is to establish an extension of (1.1), and to prove the constant factor to be the best possible. And then some important and especial results are enumerated, and an equivalent form is considered.

Received May 9, 2011.

2010 Mathematics Subject Classification. 26D15.

Key words and phrases. Hardy-Hilbert type inequality, double series, Euler-Maclaurin summation formula, weight function. 
For convenience, we introduce some notations. Let $\frac{1}{p}+\frac{1}{q}=1, p>1$ and $a=\frac{2}{p q}$. Define a function by

$$
f(x)= \begin{cases}\frac{\ln (n x)}{(n x)^{\lambda}-1}\left(\frac{n}{(n x)^{a}}\right), & \text { if } x \neq \frac{1}{n}, \\ \frac{1}{\lambda}, & \text { if } x=\frac{1}{n},\end{cases}
$$

where $n$ is a positive integer, $\lambda>1-a$ and $x \in(0,+\infty)$. It is easy to verify that $f(x)$ is continuous in $(0,+\infty)$. We stipulate that $\left\{a_{n}\right\}$ and $\left\{b_{n}\right\}$ be two non-negative sequences of real numbers. These notations will be used frequently throughout the paper.

\section{Some lemmas}

In order to prove our main results, we need the following lemmas.

Lemma 2.1. Let $f(x)$ be a function by (1.3). Then $f(x) \downarrow 0$.

Proof. Taking the derivative for $f(x)$, it is easy to deduce that

$$
f^{\prime}(x) \leq 0 \text {. }
$$

Hence the result is obtained at once.

Lemma 2.2. If substituting $y$ for $n$ in (1.3), define a function by

$$
g(y)=\frac{y \ln (x y)}{(x y)^{a}\left((x y)^{\lambda}-1\right)},
$$

then when $y$ is sufficiently large, $g(y) \downarrow 0$.

Proof. Taking the derivative for $g(y)$, it is easy to deduce that $g^{\prime}(y)<0$, when $y$ is sufficiently large. It follows that lemma is true.

Lemma 2.3. Let $0<b<1$. Then

$$
\int_{0}^{\infty} \frac{\ln u}{u-1}\left(\frac{1}{u}\right)^{b} \mathrm{~d} u=\left(\frac{\pi}{\sin (b \pi)}\right)^{2} .
$$

Proof. Let $\operatorname{Re} \alpha>\operatorname{Re} \beta>0$. It is known from the paper [5] and [7] that

$$
\int_{-\infty}^{\infty} \frac{x e^{\beta x}}{e^{\alpha x}-1} \mathrm{dx}=\left(\frac{\pi}{\alpha \sin \frac{\beta \pi}{\alpha}}\right)^{2} .
$$

Substituting $e^{t}$ for $u$ in (2.3), it is easy to deduce that

$$
\begin{aligned}
\int_{0}^{\infty} \frac{\ln u}{u-1}\left(\frac{1}{u}\right)^{b} \mathrm{du} & =\int_{-\infty}^{+\infty} \frac{t e^{(1-b) t}}{e^{t}-1} \mathrm{dt} \\
& =\left(\frac{\pi}{\sin (1-b) \pi}\right)^{2}=\left(\frac{\pi}{\sin (b \pi)}\right)^{2}
\end{aligned}
$$


Lemma 2.4. Let $n$ be a positive integer. Define a function by

$$
\omega(\lambda, n)=\sum_{m=1}^{\infty} \frac{\ln m n}{(m n)^{\lambda}-1}\left(\frac{n}{(m n)^{a}}\right) .
$$

Then

$$
\omega(\lambda, n) \leq C_{\lambda}^{2}
$$

where $C_{\lambda}$ is defined by

$$
C_{\lambda}=\frac{\pi}{\lambda \sin \left(\frac{1}{\lambda}(1-a) \pi\right)} .
$$

Proof. It is known from Lemma 2.1 that $f(x) \downarrow 0$. Hence we have

$$
\begin{aligned}
\omega(\lambda, n) & =\sum_{m=1}^{\infty} \frac{\ln m n}{(m n)^{\lambda}-1}\left(\frac{n}{(n m)^{a}}\right) \\
& \leq \int_{0}^{\infty} f(x) \mathrm{dx} \\
& =\int_{0}^{\infty} \frac{\ln n x}{(n x)^{\lambda}-1}\left(\frac{n}{(n x)^{a}}\right) \mathrm{dx} .
\end{aligned}
$$

Substituting $u$ for $(n x)^{\lambda}$, and noticing that $a=\frac{2}{p q} \leq \frac{1}{2}$. In fact, based on AG inequality we have $\sqrt{\frac{1}{p q}} \leq \frac{1}{2}\left(\frac{1}{p}+\frac{1}{q}\right)=\frac{1}{2}$. And owing to $\lambda>1-a$, we can obtain $1-\frac{1}{\lambda}(1-a)>0$. And then using (2.3), it is easy to deduce that

$$
\begin{aligned}
\int_{0}^{\infty} \frac{\ln n x}{(n x)^{\lambda}-1}\left(\frac{n}{(n x)^{a}}\right) \mathrm{dx} & =\frac{1}{\lambda^{2}} \int_{0}^{\infty} \frac{\ln u}{u-1}\left(\frac{1}{u}\right)^{1-\frac{1}{\lambda}(1-a)} \mathrm{du} \\
& =\left(\frac{\pi}{\lambda \sin \left(\frac{1}{\lambda}(1-a) \pi\right)}\right)^{2}=C_{\lambda}^{2}
\end{aligned}
$$

It follows that the inequality (2.5) is valid.

Lemma 2.5. Let $f(x)$ be a function by (1.3). Define a function by

$$
\varphi(n)=\int_{0}^{1} f(x) \mathrm{dx}-\frac{1}{2} f(1)-R(n),
$$

where $R(n)=\int_{1}^{\infty} \rho(x) f^{\prime}(x) \mathrm{dx}$ and that $\rho(x)=\{x\}-\frac{1}{2}$. Then

$$
\varphi(n)>0 \text { and } \varphi(n) \rightarrow 0 \quad(n \rightarrow \infty) .
$$

Proof. Applying Euler-Maclaurin summation formula to $\omega(\lambda, n)$ and Lemma 2.4 , we have

$$
\begin{aligned}
\omega(\lambda, n) & =\int_{1}^{\infty} f(x) \mathrm{dx}+\frac{1}{2} f(1)+\int_{1}^{\infty} \rho(x) f^{\prime}(x) \mathrm{dx} \\
& =\int_{0}^{\infty} f(x) \mathrm{d} \mathrm{x}-\varphi(n)=C_{\lambda}^{2}-\varphi(n),
\end{aligned}
$$


where $\varphi(n)$ is a function defined by $(2.7)$.

At first, based on (2.5) and (2.8), we obtain $\varphi(n)>0$.

Next, it follows from Lemma 2.2 that the integral $\int_{0}^{1} f(x) \mathrm{dx} \rightarrow 0(n \rightarrow \infty)$. Clearly $f(1)>0$ and $f(1) \rightarrow 0 \quad(n \rightarrow \infty)$.

It is known from $(2.1)$ that $f^{\prime}(x)<0$, and it is obvious that $\rho(x) \leq 0$. So we have $R(n) \geq 0$. It follows that $\varphi(n) \rightarrow 0(n \rightarrow \infty)$.

\section{Statement of main results}

Theorem 3.1. Let $\left\{a_{n}\right\}$ and $\left\{b_{n}\right\}$ be two non-negative sequences of real numbers, $\frac{1}{p}+\frac{1}{q}=1, p>1$ and $\lambda>1-a$. If $\sum_{n=1}^{\infty} n^{1-\frac{2}{p}} a_{n}^{p}<+\infty$ and $\sum_{n=1}^{\infty} n^{1-\frac{2}{q}} b_{n}^{q}<+\infty$, then

$$
\sum_{m=1}^{\infty} \sum_{n=1}^{\infty} \frac{(\ln m n) a_{m} b_{n}}{(m n)^{\lambda}-1} \leq C_{\lambda}^{2}\left(\sum_{n=1}^{\infty} n^{1-\frac{2}{p}} a_{n}^{p}\right)^{\frac{1}{p}}\left(\sum_{n=1}^{\infty} n^{1-\frac{2}{q}} b_{n}^{q}\right)^{\frac{1}{q}},
$$

where $C_{\lambda}$ is defined by (2.6). And the constant factor $C_{\lambda}^{2}$ in (3.1) is the best possible. And that the equality in (3.1) holds if and only if $\left\{a_{n}\right\}$, or $\left\{b_{n}\right\}$ is a zero-sequence.

When $\lambda=1, C_{1}$ is denoted by $C$. It is known from (2.6) that $C=\frac{\pi}{\sin (a \pi)}$.

Based on Theorem 3.1, the following result is obtained.

Corollary 3.1. With the assumptions as Theorem 3.1, then

$$
\sum_{m=1}^{\infty} \sum_{n=1}^{\infty} \frac{(\ln m n) a_{m} b_{n}}{m n-1} \leq\left(\frac{\pi}{\sin (a \pi)}\right)^{2}\left(\sum_{n=1}^{\infty} n^{1-\frac{2}{p}} a_{n}^{p}\right)^{\frac{1}{p}}\left(\sum_{n=1}^{\infty} n^{1-\frac{2}{q}} b_{n}^{q}\right)^{\frac{1}{q}},
$$

where the constant factor $\left(\frac{\pi}{\sin (a \pi)}\right)^{2}$ in (3.2) is the best possible. And that the equality in (3.2) holds if and only if $\left\{a_{n}\right\}$, or $\left\{b_{n}\right\}$ is a zero-sequence.

In particular, when $p=2$, a Hilbert-type inequality is gotten.

Corollary 3.2. If $\sum_{n=1}^{\infty} a_{n}^{2}<+\infty$ and $\sum_{n=1}^{\infty} b_{n}^{2}<+\infty$, then

$$
\sum_{m=1}^{\infty} \sum_{n=1}^{\infty} \frac{(\ln m n) a_{m} b_{n}}{(m n)^{\lambda}-1} \leq\left(\frac{\pi}{\lambda \sin \frac{\pi}{2 \lambda}}\right)^{2}\left(\sum_{n=1}^{\infty} a_{n}^{2}\right)^{\frac{1}{2}}\left(\sum_{n=1}^{\infty} b_{n}^{2}\right)^{\frac{1}{2}}
$$

where the constant factor $\left(\frac{\pi}{\lambda \sin \frac{\pi}{2 \lambda}}\right)^{2}$ in (3.3) is the best possible. And that the equality in (3.3) holds if and only if $\left\{a_{n}\right\}$, or $\left\{b_{n}\right\}$ is a zero-sequence.

If $p=2$ and $\lambda=1$, then the inequality (3.3) is reduced into the following result. 
Corollary 3.3. If $\sum_{n=1}^{\infty} a_{n}^{2}<+\infty$ and $\sum_{n=1}^{\infty} b_{n}^{2}<+\infty$, then

$$
\sum_{m=1}^{\infty} \sum_{n=1}^{\infty} \frac{(\ln m n) a_{m} b_{n}}{m n-1} \leq \pi^{2}\left(\sum_{n=1}^{\infty} a_{n}^{2}\right)^{\frac{1}{2}}\left(\sum_{n=1}^{\infty} b_{n}^{2}\right)^{\frac{1}{2}}
$$

where the constant factor $\pi^{2}$ in (3.4) is the best possible. And that the equality in (3.4) holds if and only if $\left\{a_{n}\right\}$, or $\left\{b_{n}\right\}$ is a zero-sequence.

\section{Proof of main result}

In the third section, some important results are enumerated, we only need to prove Theorem 3.1 below.

Proof of Theorem 3.1. We apply the method of the paper [9] and the Hölder inequality to estimate the left-hand side of (3.1) as follows

$$
\begin{aligned}
& \sum_{m=1}^{\infty} \sum_{n=1}^{\infty} \frac{(\ln m n) a_{m} b_{n}}{(m n)^{\lambda}-1} \\
= & \sum_{m=1}^{\infty} \sum_{n=1}^{\infty}\left(\frac{\ln m n}{(m n)^{\lambda}-1}\right)^{\frac{1}{p}}\left(\frac{m^{a / q}}{n^{a / p}} a_{m}\right)\left(\frac{\ln m n}{(m n)^{\lambda}-1}\right)^{\frac{1}{q}}\left(\frac{n^{a / p}}{m^{a / q}} b_{n}\right) \\
\leq & \left\{\sum_{m=1}^{\infty} \sum_{n=1}^{\infty} \frac{\ln m n}{(m n)^{\lambda}-1}\left(\frac{m^{a(p-1)}}{n^{a}}\right) a_{m}^{p}\right\}^{\frac{1}{p}} \\
& \left\{\sum_{m=1}^{\infty} \sum_{n=1}^{\infty} \frac{\ln m n}{(m n)^{\lambda}-1}\left(\frac{n^{a(q-1)}}{m^{a}}\right) b_{n}^{q}\right\}^{\frac{1}{q}} \\
= & \left\{\sum_{m=1}^{\infty} \omega(\lambda, m) m^{a p-1} a_{m}^{p}\right\}^{\frac{1}{p}}\left\{\sum_{n=1}^{\infty} \omega(\lambda, n) n^{a q-1} b_{n}^{q}\right\}^{\frac{1}{q}} \\
= & \left\{\sum_{n=1}^{\infty} \omega(\lambda, n) n^{1-\frac{2}{p}} a_{n}^{p}\right\}^{\frac{1}{p}}\left\{\sum_{n=1}^{\infty} \omega(\lambda, n) n^{1-\frac{2}{q}} b_{n}^{q}\right\}^{\frac{1}{q}},
\end{aligned}
$$

where the weight function $\omega(\lambda, n)$ is defined by (2.4). It follows from (2.5) and (4.2) that the inequality (3.1) is valid.

If $\left\{a_{n}\right\}$, or $\left\{b_{n}\right\}$ is a zero-sequence, then the equality in (3.1) obviously holds.

If neither $\left\{a_{n}\right\}$ nor $\left\{b_{n}\right\}$ is zero-sequences, then $0<\sum_{n=1}^{\infty} n^{1-\frac{2}{p}} a_{n}^{p}<+\infty$ and $0<\sum_{n=1}^{\infty} n^{1-\frac{2}{q}} b_{n}^{q}<+\infty$.

If (4.1) takes the form of the equality, then there exist a pair of non-zero constants $c_{1}$ and $c_{2}$ such that

$$
c_{1} \frac{\ln n m}{(n m)^{\lambda}-1} a_{m}^{p}\left(\frac{m^{a(p-1)}}{n^{a}}\right)=c_{2} \frac{\ln n m}{(n m)^{\lambda}-1} b_{n}^{q}\left(\frac{n^{a(q-1)}}{m^{a}}\right) .
$$


Hence there exists a non-zero constant $c_{0}$ such that

$$
c_{1} m^{\frac{2}{q}-1} m a_{m}^{p}=c_{2} n^{\frac{2}{p}-1} n b_{n}^{q}=c_{0} .
$$

Without loss of generality, we suppose that $c_{1} \neq 0$, then

$$
m^{\frac{2}{q}-1} m a_{m}^{p}=m^{1-\frac{2}{p}} m a_{m}^{p}=\frac{c_{0}}{c_{1}} .
$$

Hence we have

$$
\sum_{m=1}^{\infty} m^{\frac{2}{q}-1} a_{m}^{p}=\sum_{n=1}^{\infty} n^{1-\frac{2}{p}} a_{n}^{p}=\frac{c_{0}}{c_{1}} \sum_{n=1}^{\infty} \frac{1}{n} .
$$

This contradicts that $0<\sum_{n=1}^{\infty} n^{1-\frac{2}{p}} a_{n}^{p}<+\infty$. So it is impossible to take the equality in (4.1). It shows that it is also impossible to take the equality in (3.1), if neither $\left\{a_{n}\right\}$ nor $\left\{b_{n}\right\}$ is zero-sequences.

It remains to need only to show that the constant factor $C_{\lambda}^{2}$ in (3.1) is the best possible.

For arbitrarily small $\varepsilon>0$, define two sequences by $\tilde{a}_{m}=m^{-\frac{2+q \varepsilon}{p q}}$ and $\tilde{b}_{n}=n^{-\frac{2+p \varepsilon}{p q}}$. Since the sequence $\left\{\tilde{a}_{m}\right\}$ is monotonously decreasing, we have

$$
\begin{aligned}
\frac{1}{\varepsilon} & =\int_{1}^{\infty} x^{-1-\varepsilon} \mathrm{dx}<\sum_{m=1}^{\infty} m^{-1-\varepsilon}=\sum_{m=1}^{\infty} m^{1-\frac{2}{p}} m^{p\left(-\frac{2}{p q}-\frac{\varepsilon}{p}\right)} \\
& =1+\sum_{m=2}^{\infty} m^{1-\frac{2}{p}} \tilde{a}_{m}^{p}<1+\int_{1}^{\infty} x^{-1-\varepsilon} \mathrm{dx}=1+\frac{1}{\varepsilon} .
\end{aligned}
$$

Similarly,

Hence we can write

$$
\frac{1}{\varepsilon}<\sum_{n=1}^{\infty} n^{1-\frac{2}{q}} \tilde{b}_{n}^{q}<1+\frac{1}{\varepsilon}
$$

$$
\sum_{m=1}^{\infty} m^{1-\frac{2}{p}} \tilde{a}_{m}^{p}=\frac{1}{\varepsilon}+o(1) \text { and } \sum_{n=1}^{\infty} n^{1-\frac{2}{q}} \tilde{b}_{n}^{2}=\frac{1}{\varepsilon}+o(1) \quad(\varepsilon \rightarrow 0) .
$$

If $C_{\lambda}^{2}$ is not the best possible, then there exists a constant $C>0$ such that $C<C_{\lambda}^{2}$, and

$$
\begin{aligned}
S(\tilde{a}, \tilde{b})=\sum_{m=1}^{\infty} \sum_{n=1}^{\infty} \frac{(\ln m n) \tilde{a}_{m} \tilde{b}_{n}}{(m n)^{\lambda}-1} & \leq C\left(\sum_{m=1}^{\infty} m^{1-\frac{2}{p}} \tilde{a}_{m}^{p}\right)^{\frac{1}{2}}\left(\sum_{n=1}^{\infty} n^{1-\frac{2}{q}} \tilde{b}_{n}^{q}\right)^{\frac{1}{2}} \\
& =\frac{C}{\varepsilon}\{1+\circ(1)\} \quad(\varepsilon \rightarrow 0) .
\end{aligned}
$$

On the other hand, without loss of generality, we may assume that $p \geq q>1$ and $r=\frac{1}{q}-\frac{1}{p}$. It is obvious that $0 \leq r<1$. Hence we have

$$
S(\tilde{a}, \tilde{b})=\sum_{m=1}^{\infty} \sum_{n=1}^{\infty} \frac{(\ln m n) m^{-\frac{2+q \varepsilon}{p q}} n^{-\frac{2+p \varepsilon}{p q}}}{(m n)^{\lambda}-1}
$$




$$
\begin{aligned}
& =\sum_{n=1}^{\infty}\left(\sum_{m=1}^{\infty} \frac{(\ln m n)}{(m n)^{\lambda}-1} m^{-\frac{2+q \varepsilon}{p q}}\right) n^{-\frac{2+p \varepsilon}{p q}} \\
& =\sum_{n=1}^{\infty}\left(\sum_{m=1}^{\infty} \frac{(\ln m n)}{(m n)^{\lambda}-1}\left(\frac{n}{(m n)^{\left(a+\frac{\varepsilon}{p}\right)}}\right)\right) n^{-1-r \varepsilon} \\
& >\sum_{n=1}^{\infty}\left(\sum_{m=1}^{\infty} \frac{(\ln m n)}{(m n)^{\lambda}-1}\left(\frac{n}{(m n)^{\left(a+\frac{\varepsilon}{p}\right)}}\right)\right) n^{-1-\varepsilon} .
\end{aligned}
$$

When $\varepsilon$ is sufficiently small, it is known from (2.4) that

$$
\begin{aligned}
\sum_{n=1}^{\infty} \frac{(\ln m n)}{(m n)^{\lambda}-1}\left(\frac{m}{(m n)^{a+\frac{\varepsilon}{p}}}\right) & =\sum_{m=1}^{\infty} \frac{(\ln m n)}{(m n)^{\lambda}-1}\left(\frac{n}{(m n)^{a+\frac{\varepsilon}{p}}}\right) \\
& =\omega(\lambda, n)+\tilde{o}(1) \quad(\varepsilon \rightarrow 0) .
\end{aligned}
$$

It follows from (2.8), (4.4) and (4.5) that

$$
\begin{aligned}
S(\tilde{a}, \tilde{b}) & >\sum_{n=1}^{\infty}(\omega(\lambda, n)+\tilde{o}(1)) n^{-1-\varepsilon}=\sum_{n=1}^{\infty}\left\{C_{\lambda}^{2}-(\varphi(n)-\tilde{o}(1))\right\} n^{-1-\varepsilon} \\
& =C_{\lambda}^{2} \sum_{n=1}^{\infty} n^{-1-\varepsilon}-\sum_{n=1}^{\infty}(\varphi(n)-\tilde{o}(1)) n^{-1-\varepsilon} .
\end{aligned}
$$

Because the sequence $\left\{n^{-1-\varepsilon}\right\}$ is monotonously decreasing, it follows from (4.6) that

$$
\begin{aligned}
S(\tilde{a}, \tilde{b}) & >C_{\lambda}^{2} \int_{1}^{\infty} x^{-1-\varepsilon} \mathrm{dx}-\sum_{n=1}^{\infty}(\varphi(n)-\tilde{o}(1)) n^{-1-\varepsilon} \\
& =\frac{C_{\lambda}^{2}}{\varepsilon}-\sum_{n=1}^{\infty}(\varphi(n)-\tilde{o}(1)) n^{-1-\varepsilon} \quad(\varepsilon \rightarrow 0) .
\end{aligned}
$$

Below, we will show that the series $\sum_{n=1}^{\infty}(\varphi(n)-\tilde{o}(1)) n^{-1-\varepsilon}$ is bounded. In fact, it is known from Lemma 2.5 that $\varphi(n) \rightarrow 0(n \rightarrow \infty)$. Therefore there exists a positive integer $n_{0}$ such that $|\varphi(n)-\tilde{o}(1)|<\varepsilon$, when $n>n_{0}$. Consequently, we have

$$
\begin{aligned}
& \sum_{n=1}^{\infty}(\varphi(n)-\tilde{o}(1)) n^{-1-\varepsilon} \\
= & \sum_{n=1}^{n_{0}}(\varphi(n)-\tilde{o}(1)) n^{-1-\varepsilon}+\sum_{n=n_{0}+1}^{\infty}(\varphi(n)-\tilde{o}(1)) n^{-1-\varepsilon} \\
< & \sum_{n=1}^{n_{0}}(\varphi(n)-\tilde{o}(1)) n^{-1-\varepsilon}+\sum_{n=n_{0}+1}^{\infty} \varepsilon n^{-1-\varepsilon} \\
< & \sum_{n=1}^{n_{0}}(\varphi(n)-\tilde{o}(1)) n^{-1-\varepsilon}+\varepsilon \int_{n_{0}}^{\infty} x^{-1-\varepsilon} \mathrm{dx}
\end{aligned}
$$




$$
=\sum_{n=1}^{n_{0}}(\varphi(n)-\tilde{o}(1)) n^{-1-\varepsilon}+\frac{1}{n_{0}^{\varepsilon}}(\varepsilon \rightarrow 0) .
$$

It shows that the series $\sum_{n=1}^{\infty}(\varphi(n)-\tilde{o}(1)) n^{-1-\varepsilon}$ is bounded. Based on (4.7) and (4.8), we obtain

$$
S(\tilde{a}, \tilde{b})>\frac{C_{\lambda}^{2}}{\varepsilon}-O(1)(\varepsilon \rightarrow 0) .
$$

Therefore when $n$ is sufficiently large and $\varepsilon$ is sufficiently small, it follows from (4.9) that

$$
S(\tilde{a}, \tilde{b})>\frac{C_{\lambda}^{2}}{\varepsilon}(1-\circ(1)) \quad(n \rightarrow \infty \text { and } \varepsilon \rightarrow 0) .
$$

The inequality (4.10) contradicts the inequality (4.3). This shows the constant factor $C_{\lambda}^{2}$ in (3.1) is the best possible. Thus the proof of theorem is completed.

\section{Applications}

As application, we will build an equivalent form of (3.1).

Theorem 5.1. Let $\frac{1}{p}+\frac{1}{q}=1, p>1$ and $\lambda>1-a$. If $\sum_{n=1}^{\infty} n^{1-\frac{2}{p}} a_{n}^{p}<+\infty$, then

$$
\sum_{n=1}^{\infty} n^{\left(1-\frac{2}{p}\right)(p-1)}\left\{\sum_{m=1}^{\infty} \frac{\ln (m n)}{(m n)^{\lambda}-1} a_{m}\right\}^{p} \leq C_{\lambda}^{2 p} \sum_{n=1}^{\infty} n^{1-\frac{2}{p}} a_{n}^{p},
$$

where $C_{\lambda}$ is defined by (2.6). And the constant factor $C_{\lambda}^{2 p}$ in (5.1) is the best possible. And the equality in (5.1) holds if and only if $\left\{a_{n}\right\}$ is a zero-sequence. And inequality (5.1) is equivalent to (3.1).

Proof. Let $b_{n}=\left(n^{\left(1-\frac{2}{p}\right)} \sum_{m=1}^{\infty} \frac{\ln (m n)}{(m n)^{\lambda}-1} a_{m}\right)^{p-1}$. Then by (3.1), we have

$$
\begin{aligned}
& \sum_{n=1}^{\infty} n^{\left(1-\frac{2}{p}\right)(p-1)}\left\{\sum_{m=1}^{\infty} \frac{\ln (m n)}{(m n)^{\lambda}-1} a_{m}\right\}^{p} \\
= & \sum_{m=1}^{\infty} \sum_{n=1}^{\infty} \frac{\ln (m n)}{(m n)^{\lambda}-1} a_{m} b_{n} \\
\leq & C_{\lambda}^{2}\left(\sum_{n=1}^{\infty} n^{1-\frac{2}{p}} a_{n}^{p}\right)^{\frac{1}{p}}\left(\sum_{n=1}^{\infty} n^{1-\frac{2}{q}} b_{n}^{q}\right)^{\frac{1}{q}} \\
(5.2)= & C_{\lambda}^{2}\left(\sum_{n=1}^{\infty} n^{1-\frac{2}{p}} a_{n}^{p}\right)^{\frac{1}{p}}\left\{\sum_{n=1}^{\infty} n^{\left(1-\frac{2}{p}\right)(p-1)}\left(\sum_{m=1}^{\infty} \frac{\ln (m n)}{(m n)^{\lambda}-1} a_{m}\right)^{p}\right\}^{\frac{1}{q}} .
\end{aligned}
$$

The inequality (5.1) follows from (5.2) after some simplifications. 
On the other hand, assume that the inequality (5.1) is valid. Apply in turn the Hölder inequality and (5.1), we have

$$
\begin{aligned}
& \sum_{m=1}^{\infty} \sum_{n=1}^{\infty} \frac{\ln (m n)}{(m n)^{\lambda}-1} a_{m} b_{n} \\
= & \sum_{n=1}^{\infty}\left\{\left(n^{\frac{1}{p}\left(1-\frac{2}{p}\right)(p-1)} \sum_{m=1}^{\infty} \frac{\ln (m n)}{(m n)^{\lambda}-1} a_{m}\right)\left(n^{-\frac{1}{p}\left(1-\frac{2}{p}\right)(p-1)} b_{n}\right)\right\} \\
\leq & \left\{\sum_{n=1}^{\infty} n^{\left(1-\frac{2}{p}\right)(p-1)}\left(\sum_{m=1}^{\infty} \frac{\ln (m n)}{(m n)^{\lambda}-1} a_{m}\right)^{p}\right\}^{\frac{1}{p}}\left\{\sum_{n=1}^{\infty} n^{\frac{2}{p}-1} b_{n}^{q}\right\}^{\frac{1}{q}} \\
\leq & \left(C_{\lambda}^{2 p} \sum_{n=1}^{\infty} n^{1-\frac{2}{p}} a_{n}^{p}\right)^{\frac{1}{p}}\left(\sum_{n=1}^{\infty} n^{1-\frac{2}{q}} b_{n}^{q}\right)^{\frac{1}{q}} \\
= & C_{\lambda}^{2}\left(\sum_{n=1}^{\infty} n^{1-\frac{2}{p}} a_{n}^{p}\right)^{\frac{1}{p}}\left(\sum_{n=1}^{\infty} n^{1-\frac{2}{q}} b_{n}^{q}\right)^{\frac{1}{q}} .
\end{aligned}
$$

If the constant factor $C_{\lambda}^{2 p}$ in (5.1) is not the best possible, then it is known from (5.3) that the constant factor $C_{\lambda}^{2}$ in (3.1) is also not the best possible, this is in contradiction. Evidently, the equality in (5.1) holds if and only if $\left\{a_{n}\right\}$ is a zero-sequence. Consequently, the inequality (5.1) is equivalent to (3.1). The proof of theorem is completed.

Similarly, we can establish also some new inequalities which they are respectively equivalent to the inequalities (3.2), (3.3) and (3.4). These are omitted here.

Acknowledgements. The research is supported by Scientific Research Fund of Hunan Provincial Education Department (11C1045).

\section{References}

[1] M. Gao, On Hilbert's inequality and its applications, J. Math. Anal. Appl. 22 (1997), no. $1,316-323$.

[2] _ A supremum on Hardy-Hilbert's inequality with weight, J. Math. Study 31 (1998), no. 1, 18-23.

[3] M. Gao and L. Hsu, A survey of various refinements and generalizations of Hilbert's inequalities, J. Math. Res. Exposition 25 (2005), no. 2, 227-243.

[4] M. Gao and B. Yang, On the extended Hilbert's inequality, Proc. Amer. Math. Soc. 126 (1998), no. 3, 751-759.

[5] I. S. Gradshteyn and I. M. Ryzhik, Table of Integrals, Series, and Products, Academic Press, 2000.

[6] G. H. Hardy, J. E. Littlewood, and G. Polya, Inequalities, Cambridge, Cambridge Univ. Press, 1952.

[7] Y. M. Jin, Table of Applied Integrals, Hefei, University of Science and Technology of China Press, 2006.

[8] J. Kuang and L. Debnath, On new generalizations of Hilbert's inequality and their applications, J. Math. Anal. Appl. 245 (2000), no. 1, 248-265. 
[9] B. Yang, The Norm of Operator and Hilbert-Type Inequalities, Beijing, Science Press, 2009 .

Yu ZHOU

Department of Mathematics and Computer Science

Normal College, Jishou University

Jishou Hunan 416000, P. R. China

E-mail address: yuzhou863@126.com

Mingzhe Gao

Department of Mathematics and Computer Science

Normal College, Jishou University

Jishou Hunan 416000, P. R. China

E-mail address: mingzhegao@163.com 